\section{Correlation between the Amount of Clothing a Person is Wearing while Sustaining a Ballistic Injury and Wound Infection Rates}

\section{Abstract}

Introduction: The extremities are the most common area of the body to be injured from gunshot wounds (GSWs). If there are no vascular or orthopedic injuries, an operation is typically not indicated. As a bullet travels through tissue, it not only creates a cavity but also creates a vacuum both as it enters and exits. This vacuum allows debris to enter the tissue cavity and contaminate the wound. We hypothesize that a non-operative wound caused by a bullet traveling through layers of clothing will have a higher infection rate than a wound caused by a bullet that did not travel through clothing.

Methods: Patients who suffered a GSW between the years 1992 and 2017 were identified with the institutional trauma registrar at our level II rural trauma center. Patients included were those injured within our seasonal time periods; winter (November, December, January and February) and summer (May, June, July and August). Patients who did not fall into these seasonal categories, had incomplete chart documentation or failed to follow-up were excluded. Time of year was used as a surrogate for how much clothing a person was wearing when they were injured. Chart reviews were performed to identify all non-operative extremity wounds and infection rates within 30 days.

Results: A total of 233 patients were initially captured with 133 meeting inclusion criteria. Of the 76 patients included in the winter and the 57 included in the summer, the number of non-operative extremity injuries were 11 and 10 respectively. A total of 2 infections (18\%) occurred in the winter while no infections $(0 \%)$ occurred in the summer $(p=0.48)$.

Conclusion: A higher infection rate was associated with non-operative ballistic wounds to the extremities during the winter months when patients are typically wearing more clothing compared to the summer months. This difference was not statistically significant.

Keywords: Penetrating injury; Gunshot wound; Wound infection

\author{
Rachel Lovely, Rob Behm*, \\ Gabriel Ologun, \\ Andrew Trecartin, \\ Alexander Johnston, \\ Katherine Loomba, \\ Mollie Behm, Tiffany Lau and \\ Jason Granet
}

Guthrie Clinic, Guthrie Robert Packer Hospital, Sayre, Pennsylvania, United States

\section{Corresponding author: Rob Behm \\ ” robert.behm@guthrie.org}

Guthrie Robert Packer Hospital, Sayre, Pennsylvania, United States.

Tel: $570-887-2854$

Citation: Lovely R, Behm R, Ologun G, Trecartin A, Johnston A (2018) Correlation between the Amount of Clothing a Person is Wearing while Sustaining a Ballistic Injury and Wound Infection Rates. J Univer Surg. Vol.6 No.2:10

Received: February 13, 2018; Accepted: April 02, 2018; Published: April 09, 2018

\section{Introduction}

Non-fatal firearm injuries are at the highest level today since 1995 [1]. Of these injuries, the most common non-fatal gunshot wound (GSW) involves the extremities [1]. Currently, there is no uniform consensus, or standard algorithm regarding the prevention of wound infections from non-fatal GSWs. There are some literatures demonstrating that antibiotics and surgical debridement are not necessary for GSWs [2-4]. A recent study demonstrated that patients with soft tissue injuries without an associated fracture that received a single dose of intravenous antibiotics had lower infection rates compared to patients who received no antibiotic [5]. The penetrating path of bullets, and the wound tract caused by bullets has been studied rigorously, however there is limited knowledge about GSW on clothed versus non-clothed flesh. There is lack of evidence comparing the infection rates between clothed and non-clothed patients who 
have suffered a non-fatal GSW, hence making the management and treatment options variable.

Bullets themselves are not sterile, and based on the principles of hydraulics, bullets are foreign bodies and propagate skin surface bacteria into the wound [1,6]. Furthermore, pieces of clothing can also be drawn along into the wound [7]. Non-visibly soiled clothing can potentially present a significant bioburden of bacteria [8]. Additionally, the specific textile type has been shown to promote the growth of different textile microbes [9]. In areas where seasons bring about significant climate change, variability in the coverage of extremities by clothing becomes evident.

Previous studies on ballistic injury infection rates have not focused on infection rates relationship to clothing. However, it is important to study the factor clothing places on infection rates. Clothing penetration by the bullet prior to entering the tissue has potential for influencing ballistic injury protocol, specifically the infection prophylaxis protocol. The purpose of this study is to investigate whether a non-operatively managed GSW injury caused by a bullet traveling through layers of clothing will have a higher infection rate when compared to a non-operatively managed GSW caused by a bullet that did not travel through layers of clothing.

\section{Methods}

Institutional review board approval was obtained for this study. Patients were identified utilizing the institutional trauma registrar at a Level II trauma center in Pennsylvania USA. Our population of interest were patients between the years of 1992 through 2017 who suffered a gunshot wound, 233 patients were initially identified. From this initial group of 233, 4 patients were excluded. Patients were further sorted by their month of injury. The months of November, December, January and February were classified as 'winter' and the months of May, June, July and August were classified as 'summer'. Patients falling within the other four months were excluded from the study. 74 patients were excluded based on this criterion. The time of year was used as a surrogate for the amount of clothing the patient was wearing at the time of injury. In the 'winter' group extremities were assumed to be covered with clothing while in the 'summer' group extremities were assumed to not be covered with clothing. The 'winter' group contained 90 patients and the 'summer' group contained 69 patients, patients were excluded from the groups if inadequate follow up after the initial injury was documented. Based on this criterion 14 patients were excluded from the 'winter' group and 12 patients were excluded from the 'summer' group. After the sorting process 76 patients remained in the 'winter' group and 57 remained in the 'summer' group. Yielding a total of 133 patients included in the study. A chart review was performed on each of the 133 patients and the data points of age, gender, initial Systolic Blood Pressure (SBP), Injury Severity Score (ISS), survival, location of primary injury, presence of secondary injuries/ locations, surgical treatment of the wound, follow up, presence of a wound infection and caliber of the weapon. The primary outcome of interest was in gunshot wound (GSW) to the extremities which were treated nonoperatively in both the 'winter' and 'summer' groups and how many of those wounds got infected in 30 days.

\section{Results}

Of the 133 patients meeting the inclusion criteria 76 patient (57\%) were included in the 'winter' group and 57 patients (43\%) in the 'summer'. Most of the GSW patients were male, $92 \%$ in the 'winter' group and $86 \%$ in the 'summer' group. The location of the GSWs also varied between the two groups, extremity GSWs made up 51\% in the winter group and only $39 \%$ in the summer months (Figures 1 and 2). In the 'winter' group 11 GSW were managed without surgical intervention, $18 \%$ of these GSW became infected within 30 days. However, in the summer group 10 GSW were managed without surgical intervention, $0 \%$ became infected. This data had a $\mathrm{p}=0.2$ (Table 1). Furthermore, mortality rate was significantly correlated to initial SBP and to ISS in both the summer and the winter. The percent mortality significantly increased with increasing ISS and decreasing initial SBP, this trend was found in both the 'winter' and 'summer' groups. The trend for percent mortality base on ISS in the 'winter' group had a $p<0.001$ (Tables 2 and 3). While the trend for percent mortality based on initial SBP had a $p<0.005$. Similarly, the $p$ values were the same for the 'summer' group (Tables 4 and 5 ).

\section{Winter Months Location of GSW}

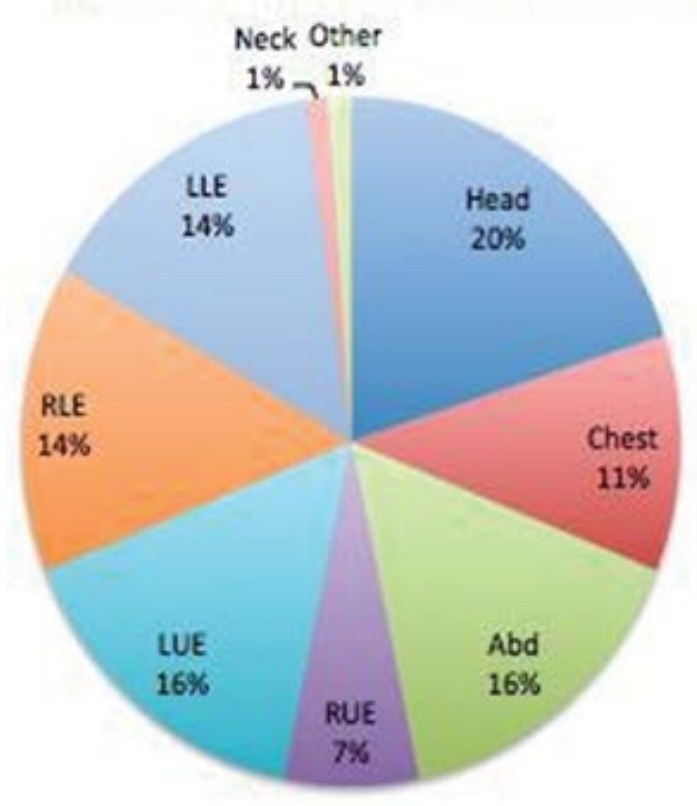

Figure 1 Location of GSW in winter months (extremity GSW makes up 51\%). RUE, right upper extremity; LUE, left upper extremity; RLE, right lower extremity; LLE, left lower extremity. 


\section{Summer Months Location of GSW}

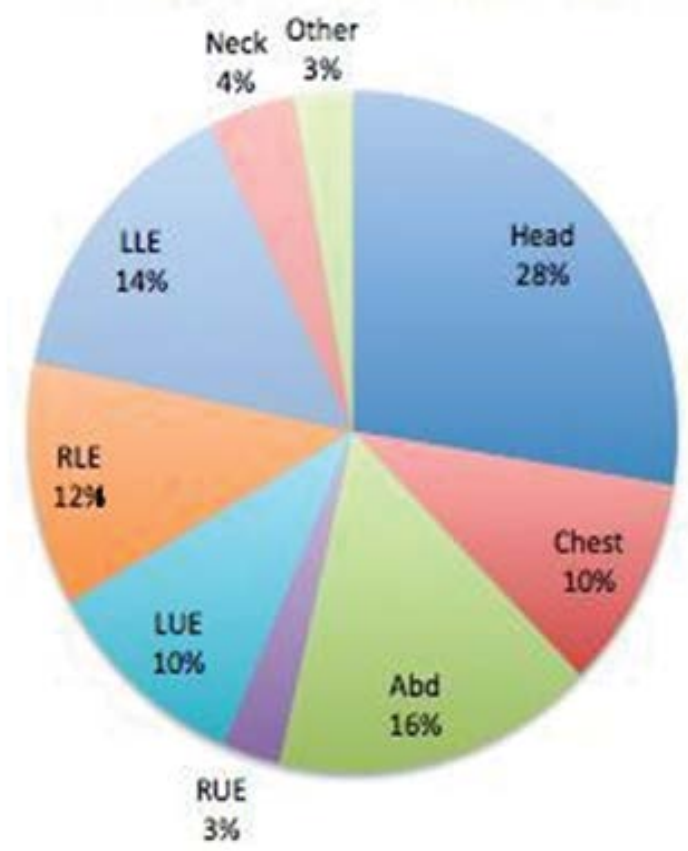

Figure 2

Location of GSW in summer months (extremity GSW makes up 39\%). RUE, right upper extremity; LUE, left upper extremity; RLE, right lower extremity; LLE, left lower extremity.

Table 1: GSW of the extremities managed non-operatively and infection rate, $p=0.2$.

\begin{tabular}{|c|c|c|c|}
\hline \multicolumn{2}{|l|}{ Winter } & \multicolumn{2}{|c|}{ Summer } \\
\hline \multicolumn{2}{|c|}{$\begin{array}{c}\text { Non operative management of } \\
\text { extremity GSW }\end{array}$} & \multicolumn{2}{|c|}{$\begin{array}{c}\text { Non operative management of } \\
\text { extremity GSW }\end{array}$} \\
\hline $\begin{array}{l}\text { Number of } \\
\text { Extremities injured }\end{array}$ & 11 & $\begin{array}{l}\text { Number of } \\
\text { Extremities injured }\end{array}$ & 10 \\
\hline Number of Infections & 2 & Number of Infections & 0 \\
\hline Infection rate & $18 \%$ & Infection rate & $0 \%$ \\
\hline
\end{tabular}

Table 2: Trends based on ISS for the winter months.

\begin{tabular}{|c|c|c|c|c|c|c|c|}
\hline & $<10$ & Oct-15 & $16-24$ & $25-34$ & $35-45$ & $>45$ & \\
\% Operations & $75 \%$ & $90 \%$ & $86 \%$ & $41 \%$ & N/A & $100 \%$ & \\
\hline \% Mortality & $0 \%$ & $0 \%$ & $14 \%$ & $41 \%$ & N/A & $50 \%$ & p $<$ \\
\% Infection & $13 \%$ & $0 \%$ & $\%$ & $18 \%$ & N/A & $0 \%$ & 0.001 \\
\hline
\end{tabular}

Table 3: Trends based on initial SBP for the winter months.

\begin{tabular}{|c|c|c|c|c|c|}
\hline & $<100$ & $100-120$ & $121-150$ & $>150$ & \\
\hline \% Operations & $63 \%$ & $69 \%$ & $80 \%$ & $69 \%$ & \\
\hline \% Mortality & $63 \%$ & $13 \%$ & $6 \%$ & $0 \%$ & $p<0.005$ \\
\hline Infection & $11 \%$ & $13 \%$ & $8 \%$ & $4.8 \%$ & \\
\hline
\end{tabular}

Table 4: Trends based on ISS for the summer months.

\begin{tabular}{|c|c|c|c|c|c|c|c|}
\hline & $<10$ & Oct-15 & $16-24$ & $25-34$ & $35-45$ & $>45$ & \\
\hline \% Operations & $67 \%$ & $67 \%$ & $100 \%$ & $33 \%$ & NIA & $0 \%$ & \\
\hline \% Mortality & $3 \%$ & $0 \%$ & $40 \%$ & $83 \%$ & N/A & $100 \%$ & p $<0.001$ \\
\hline Infection & $3 \%$ & $0 \%$ & $0 \%$ & $0 \%$ & NIA & $0 \%$ & \\
\hline
\end{tabular}

Table 5: Trends based on initial SBP for the summer months.

\begin{tabular}{|c|c|c|c|c|c|}
\hline & $<100$ & $100-120$ & $121-150$ & $>150$ & \\
\hline \% Operations & $55 \%$ & $66 \%$ & $65 \%$ & $50 \%$ & \\
\hline \% Mortal. & $77 \%$ & $25 \%$ & $15 \%$ & $6 \%$ & p $<0.005$ \\
\hline \% Infection & $0 \%$ & $0 \%$ & $5 \%$ & $0 \%$ & \\
\hline
\end{tabular}

\section{Discussion}

GSWs cause a significant number of fatalities and injuries every year [10]. In the United States firearm violence cost our country approximately \$174 billion each year [10]. Non- fatal firearm assaults are at the highest level today since 1995 [11]. The most common location of GSWs is the extremities [1]. Most low velocity GSW can be safely treated nonoperatively [1]; research is still on-going regarding the use of prophylactic antibiotics. One study observed a decreased infection rate when one dose of intravenous antibiotics was administered compared to no antibiotics administered [5].

There is currently no uniform consensus in the management of non-fatal GSWs to the extremities. There have been studies across the board demonstrating that GSWs can be safely managed without either surgical debridement or antibiotics [12]. Many GSWs to the extremities are successfully treated with nonsurgical methods. It was found that $45 \%$ to $60 \%$ of GSW patients can be treated as outpatient [1]. The management of non- fatal GSW to the extremities is continually evolving.

Clothing covering the extremities can be variable depending on the climate, especially in regions where the climate changes significantly with the seasons. Visibly clean clothing laundered in home washing machines and by professional cleaners still have significant bioburden $[8,13]$. Through ballistics research there have been significant evidence that bullets are not sterilized by the firing process [6]. Bullets when fired into tissue pull the surface substances and skin surface bacteria into the wound $[1,6]$. When bullets penetrate clothing prior to penetrating tissue, pieces of clothing fibers are carried along into the wound tract [6].

We used the time of year as a surrogate for how much clothing a person was wearing when they were injured, with the assumption that in the 'winter' group clothing was covering all extremities while in the 'summer' group there was no clothing covering the extremities.

We found that of the 11 GSW managed nonoperatively in the 'winter' group two became infected in 30 days, with an infection rate of $18 \%$, whereas in the 'summer' group of the $10 \mathrm{GSWs}$ managed nonoperatively, zero patient got infected in 30 days. Although these results are intriguing, this data is not statistically significant. The $p$-value for this data is $p=0.2$. Our limited number of patients in this subgroup $(n=21)$ likely prohibited a statistical significance in this data.

Additionally, in our chart review, we noted that percent mortality significantly increased with increasing ISS and decreasing initial SBP. This trend was found in both the 'winter' and 'summer' groups. The trend for percent mortality base on ISS in the 'winter' group had a $p<0.001$. While the trend for percent mortality 
based on initial SBP had a $p<0.005$. Similarly, the $p$ values were the same for the 'summer' group.

Our study has several limitations. Our sample size was small, and thus, our study was underpowered. The study was retrospective and only utilized data from a single institution. Additionally, we could not control for the variations in treatment decision making. Furthermore, this study operated on a number of assumptions: 1) that people uniformly wear more clothing in the winter months than when compared to the summer months, 2) that the ambient temperature corresponds to the time of year, and 3) that most patients are shot in an outdoor environment. Further research into this topic would require the study to have a larger sample size, number of GSW infections managed nonoperatively, documentation of patient's attire, and knowledge of penetration

\section{References}

1 Dougherty PJ, Najibi S, Silverton C, Vaidya R (2009) Gunshot Wounds: Epidemiology, wound ballistics and soft-tissue treatment. Instr Course Lect 58: 131-139.

2 Dougherty PJ, Vaidya R, Silverton CD, Bartlettet C, Najibi S (2009) Joint and long-bone gunshot injuries. J Bone Joint Surg Am 91: 980-997.

3 Papasoulis E, Patzakis MJ, Zalavras CG (2013) Antibiotics in the treatment of low-velocity gunshot-induced fracture: A systematic literature review. Clin Orthop Relat Res 471: 3937-3944.

4 Marecek GS, Earhart JS, Gardner MJ, Davis J, Merk BR (2016) Surgeon preferences regarding antibiotic prophylaxis for ballistic fractures. Arch Orthop Trauma Surg 136: 751-754.

5 Nguyen MP, Savakus JC, O'Donnell JA, Prayson NF, Reich MS, et al. (2017) Infection rates and treatment of low-velocity extremity gunshot injuries. J Orthop Trauma 31: 326-329.

6 Santucci RA, Chang YJ (2004) Ballistics for physicians: Myths about wound ballistics and gunshot injuries. J Urol 171: 1408-1414.

7 Dicpinigaitis PA, Koval KJ, Tejwani NC, Egol KA (2006) Gunshot wounds to the extremities. Bull NYU Hosp Jt Dis 64: 139-155. of any layers of clothing prior to penetrating the skin. In addition, antibiotic usage and the wound infection rates should be investigated when comparing clothed versus unclothed extremity GSWs.

In conclusion with the increasing frequency of GSWs and the rising costs associated with firearm violence there is a need for a standardized treatment protocol. Infection after low energy extremity GSWs occur occasionally. We found a trend, although statistically insignificant, of developing a GSW infection with the GSW occurring in the winter months (clothed extremities) when compared with the summer months (non-clothed extremities). Also it was noted that there was a statistically significant trend of percent mortality increasing with increasing ISS and decreasing initial SBP in both of the queried groups.

8 Callewaert C, Nevel SV, Kerckhof FM, Granitsiotis MS, Boon N (2015) Bacterial Exchange in Household Washing Machines. Front Microbiol 6: 1381.

9 Callewaert C, De Maeseneire E, Kerckhof FM, Verliefde A, Van de Wiele T, et al. (2014) Microbial odor profile of polyester and cotton clothes after a fitness session. Appl Environ Microbiol. 80: 66116619.

10 Strong BL, Ballard SB, Braund W (2016) The American college of preventive medicine policy recommendations on reducing and preventing firearm-related injuries and deaths. Am J Prev Med 51: 1084- 1089.

11 Stefanopoulos PK, Pinialidis DE, Hadjigeorgiou GF, Filippakis K, Gyftokostas D (2015) Wound ballistics of gunshot injuries. Hellenic J Surg 87: 351-356.

12 Sathiyakumar V, Thakore RV, Stinner DJ, Obremskey WT, Ficke JR, et al. (2015) Gunshot-induced fractures of the extremities: A review of antibiotic and debridement practices. Curr Rev Musculoskelet Med 8: 276-289.

13 Oie S, Furukawa H, Kobayashi H, Okubo T (2016) Cleanliness of linen and clothing items professionally laundered or dry-cleaned. Jpn J Infect Dis 69: 75-76. 